\title{
What could a new disease activity score for polymyalgia rheumatica do better?
}

\section{Burkhard F. Leeb (D)}

In their Review on monitoring and longterm management of giant cell arteritis (GCA) and polymyalgia rheumatica (PMR) (Camellino, D., Matteson, E. L., Buttgereit, F. \& Dejaco, C. Monitoring and long-term management of giant cell arteritis and polymyalgia rheumatica. Nat. Rev. Rheumatol. 16, 481-495 (2020) $)^{1}$, Camellino et al. considered the need for a new disease activity score for PMR, concluding that the established Polymyalgia Rheumatica Activity Score (PMR-AS) is seldom used in clinical practice or in clinical trials. To date, the publication in which the PMR-AS was first proposed ${ }^{2}$ has been cited 138 times according to Google Scholar, 13 of which were in 2020 , which gives testimony to its acceptance and implementation in scientific rheumatology.

The PMR-AS was originally derived from the EULAR response criteria for $\mathrm{PMR}^{3}$. Pain was recognized as such an important feature that it was chosen as the only parameter that had to decrease obligatorily in the PMR-AS, whereas at least three of the other four features, namely the physician's global assessment (PGA), C-reactive protein (CRP) level or erythrocyte sedimentation rate (ESR), upper limb elevation and morning stiffness, had to improve.

On the basis of an OMERACT study group publication $^{4}$ from 2017, Camellino et al. proposed that systemic inflammation detected by laboratory tests, physical function, pain and stiffness should be included in a new score for PMR monitoring ${ }^{1}$. This statement might surprise rheumatologists, as the individual components of the PMR-AS already cover all those domains.

Either a CRP level or an ESR can be used to calculate the PMR-AS, both of which are the acute-phase reactant tests predominantly used in clinical practice. One can debate whether upper limb elevation is the best measure of functionality, however, it is a cardinal symptom of PMR, and morning stiffness clearly covers the stiffness parameter. Of course, a discussion of how important the PGA is for a score's reliability is justified. However, other widely used scores include the PGA, which is often designated as the counterbalance to patient-related parameters ${ }^{5,6}$.
Cronbach's alpha (a measure of reliability) for the PMR-AS was between 0.91 and 0.88 in two patient cohorts $(>0.7$ indicates high reliability), and factorial analysis showed that all five single parameters contribute considerably to the overall result, with pain and PGA exerting the greatest influence ${ }^{2}$.

The PMR-AS has been applied in several studies $^{7,8}$ and comprises all the parameters proposed by Camellino et al. ${ }^{1}$ : why then should this score not be used for monitoring PMR in clinical practice or in clinical trials, or even be used as a surrogate for remission ${ }^{9}$ ? What should a new score be capable of that the PMR-AS is not? Using other parameters for function and stiffness, or leaving out the PGA, might change something, but pain and acute-phase reactants are not interchangeable and, ultimately, a new score must achieve the high internal consistency of the PMR-AS. Would developing a new score not be like the reinvention of the wheel?

There is a reply to this letter by Camellino, $\mathrm{D}$., Matteson, E. L., Buttgereit, F. \& Dejaco, C.
Nat. Rev. Rheumatol. https://doi.org/10.1038/ s41584-020-00551-5 (2020).

\section{Burkhard F. Leeb (iD) ${ }^{1,2}$ University Clinic for Internal Medicine - Rheumatology, St. Poelten, Austria. ${ }^{2}$ Karl Landsteiner Institute for Clinical Rheumatology, Hollabrunn, Austria. e-mail: leeb.rheuma@aon.at} https://doi.org/10.1038/s41584-020-00550-62021 Feb8

. Camellino, D., Matteson, E. L., Buttgereit, F. $\ltimes$ Dejaco, C. Monitoring and long-term management of giant cell arteritis and polymyalgia rheumatica. Nat. Rev. Rheumatol. 16, 481-495 (2020).

2. Leeb, B. F. \& Bird, H. A. A disease activity score for polymyalgia rheumatica. Ann. Rheum. Dis. 63 1279-1283 (2004).

3. Leeb, B. F. et al. EULAR response criteria for polymyalgia rheumatica: results of an initiative of the European Collaborating Polymyalgia Rheumatica Group (subcommittee of ESCISIT) Ann. Rheum. Dis. 62, 1189-1194 (2003).

4. Mackie, S. L. et al. The OMERACT core domain set for outcome measures for clinical trials in polymyalgia rheumatic. J. Rheumatol. 44, 1515-1521 (2017).

5. Smolen, J. S. et al. A simplified disease activity index for rheumatoid arthritis for use in clinical practice. Rheumatology 42, 244-257 (2003).

6. Aletaha, D. et al. Acute phase reactants add little to composite disease activity indices for rheumatoid arthritis: validation of a clinical activity score. Arthritis Res. Ther. 7, R796-R806 (2005).

7. Devauchelle-Pensec, V. et al. Assessing polymyalgia rheumatica activity when C-reactive protein is unavailable or uninterpretable. Rheumatology 57 , 666-670 (2018).

8. Manzo, C. \& Natale, M. Relapse of polymyalgia rheumatica after a fall. Reumatologia 55, 251-255 (2017).

9. Nothnagl, T. \& Leeb, B. F. Diagnosis, differential diagnosis and treatment of polymyalgia rheumatica. Drugs Aging 23, 391-402 (2006).

\section{Reply to: What could a new disease activity score for polymyalgia rheumatica do better?}

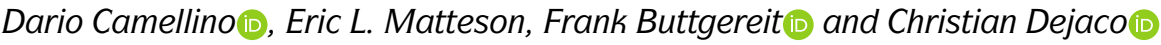

We thank Prof. Leeb for his interest in our Review (Camellino, D., Matteson, E. L., Buttgereit, F. \& Dejaco, C. Monitoring and long-term management of giant cell arteritis and polymyalgia rheumatica. Nat. Rev. Rheumatol. 16, 481-495 (2020)) $)^{1}$ and for his comments (Leeb, B. F. What could a new disease activity score for polymyalgia rheumatica do better? Nat. Rev. Rheumatol. https://doi.org/10.1038/s41584-020-00550-6 $(2020))^{2}$. As he points out, the Polymyalgia Rheumatica Activity Score (PMR-AS) is currently the only validated score for monitoring disease activity in patients with $\mathrm{PMR}^{3}$. Score calculation is straightforward, incorporating standard clinical data that captures disease features (including pain, stiffness, elevation of the upper limbs, physician's global assessment and inflammatory indexes) relevant for the evaluation of a patient with PMR.

Despite these advantages, the PMR-AS does not seem to be widely used. On 17 October 2020, we carried out a search of studies in patients with PMR through the Clinicaltrials. gov database. Among 105 studies retrieved, seven trials explicitly included the PMR-AS among their outcomes. We also analysed the publications included in the 2015 ACREULAR recommendations for the management of $\mathrm{PMR}^{4}$. Among the nine studies on 
interventions published after 2004 (the year the PMR-AS was published), only one trial reported using the score ${ }^{5}$. Similarly, a 2020 systematic review from an OMERACT special interest group ${ }^{6}$ identified 13 studies using the PMR-AS out of a total of 46 .

The value of a clinical index is not defined by the number of its users. It is well recognized that composite scores, which are the pillars of treat-to-target strategies in rheumatoid arthritis, are often overlooked in clinical practice owing to time constraints or to the belief that the overall impression of the physician could be sufficient to assess the patient's state comprehensively ${ }^{7}$.

The aim of the OMERACT initiative is to develop core sets of measures for the evaluation of patients with rheumatic diseases ${ }^{8}$. In 2017, laboratory indexes, pain, stiffness and physical function were selected as candidates for the inner core of domains that should be present in every clinical trial in $\mathrm{PMR}^{9}$. However, high-quality evidence was not available for any of the instruments currently used to measure these domains ${ }^{6}$. Physical function is one of the most critical domains for patients, but the sole assessment of upper limb elevation seems insufficient to fully describe the impairment of physical function ${ }^{6}$. In a survey of 78 patients with PMR, the experience of stiffness and the effect of fatigue were identified as themes not adequately captured by the candidate instruments, suggesting the need for new patient-reported outcome measures ${ }^{10}$. PMR is a multifaceted disease, in which different pathophysiological manifestations can co-exist and have different clinical effects on patients. Moreover, the objective evaluation of the patient's status might be further complicated by pre-existing conditions or by glucocorticoid-related adverse effects.

The PMR-AS is the first, and currently the only, instrument to monitor disease in patients with PMR. That notwithstanding, there is still a long road ahead before we have a comprehensive tool that can be used to objectively assess disease activity and that embraces patients' experiences.

Dario Camellino (iD ${ }^{1,2}$, Eric L. Matteson 3 , Frank Buttgereit (iD ${ }^{4}$ and Christian Dejaco iD ${ }^{5,6}$ 'Division of Rheumatology, Local Health Trust 3 , Genoa, Italy. ${ }^{2}$ Autoimmunology Laboratory, University of Genoa Genoa, Italy.

${ }^{3}$ Division of Rheumatology and Division of Epidemiology, Mayo Clinic College of Medicine and Science, Rochester, MN, USA. ${ }^{4}$ Department of Rheumatology and Clinical Immunology, Charité University Medicine, Berlin, Germany. ${ }^{5}$ Department of Rheumatology and Immunology, Medical University Graz, Graz, Austria. ${ }^{6}$ Department of Rheumatology, Hospital of Brunico (SABES-ASDAA), Bruneck, Italy.

凶e-mail: christian.dejaco@gmx.net https://doi.org/10.1038/s41584-020-00551-5

1. Camellino, D., Matteson, E. L., Buttgereit, F. \& Dejaco, $\mathrm{C}$. Monitoring and long-term management of giant cell arteritis and polymyalgia rheumatica. Nat. Rev. Rheumatol. 16, 481-495 (2020).
2. Leeb, B. F. What could a new disease activity score for polymyalgia rheumatica do better? Nat. Rev. Rheumatol. https://doi.org/10.1038/s41584-020. 00550-6 (2020).

3. Leeb, B. F. \& Bird, H. A. A disease activity score for polymyalgia rheumatica. Ann. Rheum. Dis. 63 1279-1283 (2004).

4. Dejaco, C. et al. 2015 recommendations for the management of polymyalgia rheumatica: A European League Against Rheumatism/American College of Rheumatology collaborative initiative. Ann. Rheum Dis. 74, 1799-1807 (2015).

5. Kreiner, F. \& Galbo, H. Effect of etanercept in polymyalgia rheumatica: a randomized controlled trial. Arthritis Res. Ther. 12, R176 (2010).

6. Twohig, H. et al. Outcomes measured in polymyalgia rheumatica and measurement properties of instruments considered for the OMERACT core outcome set: a systematic review. J. Rheumatol. https://doi.org/10.3899/jrheum.200248 (2020).

7. Lindsay, K. et al. The composite DAS Score is impractical to use in daily practice: evidence that physicians use the objective component of the DAS in decision making.

J. Clin. Rheumatol. 15, 223-225 (2009).

8. Tugwell, P. et al. OMERACT: An international initiative to improve outcome measurement in rheumatology. Trials 8, 38 (2007).

9. Mackie, S. L. et al. The OMERACT core domain set for outcome measures for clinical trials in polymyalgia rheumatica. J. Rheumatol. 44, 1515-1521 (2017).

10. Yates, M. et al. Feasibility and face validity of outcome measures for use in future studies of polymyalgia rheumatica: An OMERACT study. J. Rheumatol. 47 1379-1384 (2020).

\section{Competing interests}

D.C. declares that he has received consultancy and speaker fees from AbbVie, Celgene, Janssen-Cilag, Lilly, Mylan, Novartis and Sanofi; none of which is related to this manuscript. E.L.M. declares that he is a site investigator for the GiACTA study and a contributor and section editor to UpToDate. F.B. declares that he has received consultancy and speaker fees and research grants from Horizon, Mundipharma and Roche/Chugai; none of which is related to this manuscript. C.D. declares that he has received consultancy and speaker fees from AbbVie, BMS, Lilly, MSD, Novartis, Pfizer, Roche, Sanofi and UCB and a research grant from Celgene; none of which is related to this manuscript. 